\title{
Integrability of Discrete Equations Modulo a Prime
}

\author{
Masataka KANKI \\ Graduate School of Mathematical Sciences, \\ University of Tokyo, 3-8-1 Komaba, Tokyo 153-8914, Japan \\ E-mail:kanki@ms.u-tokyo.ac.jp
}

Received April 24, 2013, in final form September 05, 2013; Published online September 08, 2013 http://dx.doi.org/10.3842/SIGMA.2013.056

\begin{abstract}
We apply the "almost good reduction" (AGR) criterion, which has been introduced in our previous works, to several classes of discrete integrable equations. We verify our conjecture that AGR plays the same role for maps of the plane define over simple finite fields as the notion of the singularity confinement does. We first prove that $q$-discrete analogues of the Painlevé III and IV equations have AGR. We next prove that the Hietarinta-Viallet equation, a non-integrable chaotic system also has AGR.
\end{abstract}

Key words: integrability test; good reduction; discrete Painlevé equation; finite field

2010 Mathematics Subject Classification: 37K10; 34M55; 37P25

\section{Introduction}

The purpose of this paper is to define the nonlinear discrete integrable equations over finite fields and to investigate how to formulate the integrability of them over finite fields, with the help of the theory of arithmetic dynamics. In the theory of arithmetic dynamics, we are interested in how the properties of the mappings change as we change the set on which the mappings are defined [15]. In particular, the system over the field of $p$-adic integers and its reduction modulo a prime to the finite field attracts much attention. We have another interest in the dynamical systems over finite fields in terms of cellular automata, of which the underlying set consists of a finite number of elements and the mapping is given by recurrence formulae [18].

Let us first explain the problems we encounter and review some of the previous results. In the case of linear discrete equations, there is no problem in defining the equations over finite fields just by changing the field on which the equations are defined to finite fields. This approach can also be valid for equations such as bilinear equations. In this direction, the discrete KP and $\mathrm{KdV}$ equations and their soliton solutions over finite fields have been investigated [2]. However, in order to deal with nonlinear discrete equations, we frequently pass through division by zero modulo prime and indeterminacies (e.g. $0 / 0, \infty \pm \infty$ ), which prevent us from obtaining the evolution. One of the methods to tackle this difficulty is to restrict the domain of definition so that we do not need to treat indeterminacies. For example we can terminate the evolution of the equation when it hits these points. Integrability of the discrete equations over finite fields has been investigated in terms of the lengths of the periodic and terminating orbits $[12,13]$. The graph structures of the discrete Toda equation whose dependent variables are limited to non-zero values have been studied [16].

We take another approach in this paper: we extend the space of initial conditions instead of restricting it. We have two main ways of extension. The first method is to apply Sakai's theory for discrete Painlevé equations [14] over the finite field. According to this theory we can construct the birational mapping over the extended space of initial conditions by blowing up at each singular point. The space of initial conditions for the discrete Painlevé II equation has been established over $\mathbb{F}_{p}$ and the bijection between a finite number of points has been constructed [8]. 
The second approach uses the field of $p$-adic numbers. We study this method in detail from here on. We define the discrete integrable equations over the field of $p$-adic numbers $\mathbb{Q}_{p}$, and then define them over $\mathbb{F}_{p}$, so that they are compatible with the reduction modulo prime from those over $\mathbb{Q}_{p}$. Rational mappings are said to have good reduction if, roughly speaking, the reduction and the evolution of the system commute. One of the typical examples with good reduction is the fractional linear transformation related to the projective linear group $\mathrm{PGL}_{2}$. Recently, birational mappings over finite fields have been investigated in terms of integrability [12]. In the previous papers, we defined the generalized notion of good reduction so that it could be applied to wider class of integrable mappings. We called this notion "almost good reduction" (AGR), and proved that discrete and $q$-discrete Painlevé II equations have AGR [7, 8]. Our conjecture was that AGR is also satisfied for other discrete Painlevé equations and that AGR is closely related to the integrability of dynamical systems over finite fields. In this paper, we prove that several types of $q$-discrete analogues of the Painlevé equations [11] have AGR for an appropriate domain, thereby verifying the conjecture. We also study the application of AGR to a chaotic system - Hietarinta-Viallet equation [5] - and conclude that AGR can be seen as an arithmetic analogue of the singularity confinement test [3].

\section{Reduction modulo a prime}

Let $p$ be a prime number. Each non-zero rational number $x \in \mathbb{Q}^{\times}$has a unique representation $x=p^{v_{p}(x)} \frac{u}{v}$ where $v_{p}(x), u, v \in \mathbb{Z}$ and $u$ and $v$ are coprime integers neither of which is divisible by $p$. The $p$-adic norm $|x|_{p}$ is defined as $|x|_{p}:=p^{-v_{p}(x)}\left(|0|_{p}:=0\right)$. The field of $p$-adic numbers $\mathbb{Q}_{p}$ is defined as a completion of $\mathbb{Q}$ with respect to the $p$-adic norm. The ring of $p$-adic integers is defined as

$$
\mathbb{Z}_{p}:=\left\{\left.x \in \mathbb{Q}_{p}|| x\right|_{p} \leq 1\right\} .
$$

The ring $\mathbb{Z}_{p}$ has the unique maximal ideal

$$
\mathfrak{p}=p \mathbb{Z}_{p}=\left\{\left.x \in \mathbb{Z}_{p}|| x\right|_{p}<1\right\} .
$$

We define the reduction of $x$ modulo $\mathfrak{p}$ by

$$
\mathbb{Z}_{p} \ni x \mapsto(x \bmod \mathfrak{p}) \in \mathbb{Z}_{p} / \mathfrak{p} \cong \mathbb{F}_{p}
$$

and denote $(x \bmod \mathfrak{p})$ as $\tilde{x}$. The above mapping defines a reduction of $p$-adic integers to the (simple) finite field. Note that if we limit $x$ to be a (rational) integer, then $\tilde{x}$ is nothing but $x$ modulo $p$. The reduction is naturally generalised to $\mathbb{Q}_{p}$ :

$$
\mathbb{Q}_{p} \ni x \mapsto\left\{\begin{array}{ll}
\tilde{x}, & x \in \mathbb{Z}_{p}, \\
\infty, & x \in \mathbb{Q}_{p} \backslash \mathbb{Z}_{p}
\end{array} \in \mathbb{P F}_{p} .\right.
$$

We also denote the right hand side by $\tilde{x}$. Here the space $\mathbb{P F}_{p}$ denotes the projective line $\mathbb{P}^{1}\left(\mathbb{F}_{p}\right)$ defined over the finite field $\mathbb{F}_{p}$. As a set, we have $\mathbb{P F}_{p}=\mathbb{F}_{p} \cup\{\infty\}$.

Next we define the reduction of maps of the plane. Let $\phi$ be a rational map of the plane $\mathbb{Q}_{p}^{2}$ given by two rational functions defined over $(x, y) \in \mathbb{Q}_{p}^{2}$ :

$$
\phi(x, y)=(f(x, y), g(x, y)),
$$

where $f, g \in \mathbb{Q}_{p}(x, y)$ are rational functions. By multiplying numerators and denominators of $f$ and $g$ by suitable powers of $p$, the coefficients of both $f$ and $g$ can be taken in $\mathbb{Z}_{p}$ and that at least one of the coefficients is in $\mathbb{Z}_{p}^{\times}$. From here on we assume this "minimal" form for rational 
functions. If neither of the denominator of the minimal form of $f$ or that of $g$ modulo $\mathfrak{p}$ is zero, then $\tilde{\phi}$ is defined as the system whose coefficients are reduced to $\mathbb{F}_{p}$ :

$$
\tilde{\phi}(x, y)=(\tilde{f}(x, y), \tilde{g}(x, y)) \in\left(\mathbb{F}_{p}(x, y)\right)^{2},
$$

where $(x, y) \in \mathbb{Q}_{p}^{2}$. The map $\phi$ is said to have good reduction (modulo $\mathfrak{p}$ ) on the domain $\mathcal{D} \subseteq \mathbb{Z}_{p}^{2}$, if we have

$$
\widetilde{\phi(x, y)}=\tilde{\phi}(\tilde{x}, \tilde{y})
$$

for any $(x, y) \in \mathcal{D}[15]$. Although the good reduction is useful in arithmetic dynamical systems, the discrete Painlevé equations (expressed as a dynamical system) do not have good reduction since they frequently pass through singularities after reducing the equations modulo a prime. Also, the discrete Painlevé equations are non-autonomous mappings, and we need a generalization of good reduction to a non-autonomous mapping. Therefore, we have modified the good reduction so that it can be applied to wider class of systems, in particular to the discrete Painlevé equations.

Definition 2.1 ([7]). A (non-autonomous) rational map of the plane $\phi_{n}$ has almost good reduction (AGR) modulo $\mathfrak{p}$ on the domain $\mathcal{D}^{(n)} \subseteq \mathbb{Z}_{p}^{2} \cap \phi_{n}^{-1}\left(\mathbb{Q}_{p}^{2}\right)$ if for any $\mathrm{p}=(x, y) \in \mathcal{D}^{(n)}$ and any time step $n$, there exists a positive integer $m_{\mathrm{p} ; n}$ such that

$$
\widehat{\phi_{n}^{m_{\mathrm{p} ; n}}(x, y)}=\widehat{\phi_{n}^{m_{\mathrm{p} ; n}}}(\tilde{x}, \tilde{y}) \text {. }
$$

Here, the iteration $\phi_{n}^{m}$ is defined as $\phi_{n}^{m}:=\left.\left(\phi_{n+m-1} \circ \phi_{n+m-2} \circ \cdots \circ \phi_{n}\right)\right|_{\mathcal{D}^{(n)}}$ for $m>0$.

If the domain $\mathcal{D}^{(n)}$ does not depend on $n$, we just denote it by $\mathcal{D}$. Note that, in particular, if we can take $m_{\mathrm{p} ; n}=1$ for all points $\mathrm{p} \in \mathcal{D}^{(n)}$ and all $n$, then the mapping $\phi_{n}$ has good reduction. Therefore AGR is weaker than good reduction.

The following simple mapping $\Psi_{\gamma}$ illustrates how almost good reduction works. Let us define

$$
\Psi_{\gamma}:\left\{\begin{array}{l}
x_{n+1}=\frac{a x_{n}+1}{x_{n}^{\gamma} y_{n}} \\
y_{n+1}=x_{n}
\end{array}\right.
$$

where $|a|_{p} \leq 1$ and $\gamma \in \mathbb{Z}_{\geq 0}$ are parameters. Note that we omitted the cases of $|a|_{p}>1$, since we have $v_{p}(a)<0$ in this case, and we have to deal with a mapping such as

$$
\phi: x_{n+1}=\frac{\frac{x_{n}}{p}+1}{x_{n}^{\gamma} y_{n}}=\frac{x_{n}+p}{p x_{n}^{\gamma} y_{n}}, \quad y_{n+1}=x_{n},
$$

whose reduction of coefficients $\widetilde{\phi}$ is not well-defined (note that $\widetilde{1 / p}=\infty$ ). The map (1) is known to be integrable if and only if $\gamma=0,1,2$. In these cases the map is a symmetric QRT mapping [9]. We have proved in our previous work that the following proposition holds.

Proposition $2.2([7])$. The rational mapping (1) has almost good reduction modulo $\mathfrak{p}$ on the domain $\mathcal{D}$ if and only if $\gamma=0,1,2$. Here $\mathcal{D}:=\mathbb{Z}_{p}^{2} \cap \Psi_{\gamma}^{-1}\left(\mathbb{Q}_{p}^{2}\right)$. If $\gamma=1,2$ then $\mathcal{D}=\{(x, y) \in$ $\left.\mathbb{Z}_{p}^{2} \mid x \neq 0, y \neq 0\right\}$. If $\gamma=0$ then $\mathcal{D}=\left\{(x, y) \in \mathbb{Z}_{p}^{2} \mid y \neq 0\right\}$.

We have also proved that the discrete and $q$-discrete Painlevé II equations also have almost good reduction $[7,8]$. From these observations we have conjectured that, for the map of the plane $\Phi_{n}$ defined over the field of $p$-adic numbers, having almost good reduction on the domain $\mathcal{D}^{(n)}=\mathbb{Z}_{p}^{2} \cap \Phi_{n}^{-1}\left(\mathbb{Q}_{p}^{2}\right)$ is equivalent to passing the singularity confinement test [3]. In this article, we support this conjecture by presenting further applications of the almost good reduction 
principle to other integrable equations such as several types of $q$-discrete Painlevé equations and a chaotic equation.

Note that in this paper we only deal with simple finite fields $\mathbb{F}_{p}$. To study the equations over a general finite field $\mathbb{F}_{p^{m}}(m>1)$, we need to use the field extension of $\mathbb{Q}_{p}$. Since a field extension $L$ of finite degree $m$ over $\mathbb{Q}_{p}$ is a simple extension, there exists an element $\alpha \in L$ such that $L=\mathbb{Q}_{p}(\alpha)$. The reduction from $L$ to the extension of the finite field $\mathbb{F}_{p}(\alpha)$ is defined as

$$
L \ni \sum_{i=0}^{m-1} x_{i} \alpha^{i} \mapsto \begin{cases}\sum_{i=0}^{m-1} \tilde{x}_{i} \alpha^{i}, & \forall i x_{i} \in \mathbb{Z}_{p}, \\ \infty, & \exists i x_{i} \in \mathbb{Q}_{p} \backslash \mathbb{Z}_{p} .\end{cases}
$$

From $\mathbb{F}_{p}(\alpha) \cong \mathbb{F}_{p^{m}}$, we obtain a system over a general finite field. Since the $p$-adic norm of $\mathbb{Q}_{p}$ is extended to $L$, propositions in this paper are still valid for $L$, with slight modifications (e.g. $\mathcal{D}$ becomes $\left(\mathbb{Z}_{p}^{\oplus m}\right)^{2} \cap \Psi_{\gamma}^{-1}\left(L^{2}\right)$ in Proposition 2.2$)$.

\section{$3 q$-difference analogue of Painlevé equations over a finite field}

In this section we prove that the $q$-discrete analogues of Painlevé III and IV equations have almost good reduction. These two equations are indeed integrable in the sense that they pass the singularity confinement test [3]. Note that, although passing singularity confinement test is not equivalent to the integrability of the given discrete equation, singularity confinement can be seen as a discrete analogue of the Painlevé property of the continuous Painlevé equations. In the geometrical setting, these two equations have rational surfaces of initial conditions on which the equations are birational [14]. From here on we occasionally write $a \equiv b$ for $a, b \in \mathbb{Z}_{p}$, to indicate that $\tilde{a}=\tilde{b} \in \mathbb{F}_{p}$.

\section{$3.1 \quad q$-discrete Painlevé III equation}

The $q$-discrete analogue of Painlevé III equation has the following form

$$
x_{n+1} x_{n-1}=\frac{a b\left(x_{n}-c q^{n}\right)\left(x_{n}-d q^{n}\right)}{\left(x_{n}-a\right)\left(x_{n}-b\right)},
$$

where $a, b, c, d$ and $q$ are parameters [11]. It is convenient to rewrite it as the following coupled system form

$$
\Phi_{n}:\left\{\begin{array}{l}
x_{n+1}=\frac{a b\left(x_{n}-c q^{n}\right)\left(x_{n}-d q^{n}\right)}{y_{n}\left(x_{n}-a\right)\left(x_{n}-b\right)}, \\
y_{n+1}=x_{n} .
\end{array}\right.
$$

Proposition 3.1. Suppose that $a, b, c, d, q$ are distinct parameters with $|a|_{p}=|b|_{p}=|c|_{p}=$ $|d|_{p}=1$, and we also suppose that $a+b \not \equiv(c+d) q^{3}$ and $a \not \equiv b$, then the mapping (2) has almost good reduction modulo $\mathfrak{p}$ on the domain $\mathcal{D}:=\left\{(x, y) \in \mathbb{Z}_{p}^{2} \mid x \neq a, b, y \neq 0\right\}$.

Proof. Let $\left(x_{n+1}, y_{n+1}\right)=\Phi_{n}\left(x_{n}, y_{n}\right)$. In the case when $\tilde{x}_{n} \neq \tilde{a}, \tilde{b}$ and $\tilde{y}_{n} \neq 0$, we have

$$
\begin{aligned}
& \tilde{x}_{n+1}=\frac{\tilde{a} \tilde{b}\left(\tilde{x}_{n}-\tilde{c} \tilde{q}^{n}\right)\left(\tilde{x}_{n}-\tilde{d} \tilde{q}^{n}\right)}{\tilde{y}_{n}\left(\tilde{x}_{n}-\tilde{a}\right)\left(\tilde{x}_{n}-\tilde{b}\right)}, \\
& \tilde{y}_{n+1}=\tilde{x}_{n} .
\end{aligned}
$$

since the reduction modulo $p \mathbb{Z}_{p}$ is a ring homomorphism. Hence clearly $\widetilde{\Phi_{n}\left(x_{n}, y_{n}\right)}=\widetilde{\Phi_{n}}\left(\tilde{x}_{n}, \tilde{y}_{n}\right)$. Next we examine other cases. We investigate the iterated maps for every initial condition 
$\left(x_{n}, y_{n}\right) \in \mathcal{D}$. There are six cases to consider. They are essential since the behaviors around the singular points are involved. From here we sometimes abbreviate $\tilde{a}$ as $a, \tilde{b}$ as $b$ for simplicity.

(i) Let us first consider the case where

$$
x_{n} \equiv a \quad \text { and } \quad(a-b)(a+b-c q-d q) \tilde{y}_{n} \not \equiv b(a-c)(a-d) .
$$

In this case, $\widetilde{\Phi_{n}}\left(\tilde{a}, \tilde{y}_{n}\right)$ is not well-defined. This is because we have $\tilde{x}_{n}-\tilde{a}=\tilde{a}-\tilde{a}=0$ in the denominator of $\tilde{x}_{n+1}$. We also learn from $y_{n+2}=x_{n+1}$, that $\tilde{y}_{n+2}$ is not defined either. Therefore $\widetilde{\Phi_{n}^{2}}\left(\tilde{a}, \tilde{y}_{n}\right)$ is not well-defined. Here $\widetilde{\Phi_{n}^{2}}:=\widetilde{\Phi_{n+1} \circ \Phi_{n}}$.

However, at the third iteration step, $\widetilde{\Phi_{n}^{3}}\left(\tilde{a}, \tilde{y}_{n}\right)$ is well-defined and we have

$$
\widetilde{\Phi_{n}^{3}\left(x_{n}, y_{n}\right)}=\widetilde{\Phi_{n}^{3}}\left(\tilde{x}_{n}=\tilde{a}, \tilde{y}_{n}\right)=\left(\frac{a\left(b-c q^{2}\right)\left(b-d q^{2}\right) \tilde{y}_{n}}{b(a-c)(a-d)-(a-b)(a+b-c q-d q) \tilde{y}_{n}}, b\right) .
$$

From the assumption of the case (i), the denominator is nonzero and is in $\mathbb{F}_{p}^{\times}$.

(ii) Next we investigate the case of

$$
x_{n} \equiv a \quad \text { and } \quad(a-b)(a+b-c q-d q) \tilde{y}_{n} \equiv b(a-c)(a-d)
$$

In this case, none of $\widetilde{\Phi_{n}^{i}}\left(\tilde{a}, \tilde{y}_{n}\right)$ is well-defined for $i=1,2,3,4$. Next we calculate the fifth iteration $\Phi_{n}^{5}$ at $\tilde{y}_{n} \equiv \frac{b(a-c)(a-d)}{(a-b)(a+b-c q-d q)}$ and simplify the outcome. Then we learn that $\widetilde{\Phi_{n}^{5}}\left(\tilde{a}, \tilde{y}_{n}\right)$ is well-defined and we have

$$
\Phi_{n}^{5} \widetilde{\left(x_{n}, y_{n}\right)}=\widetilde{\Phi_{n}^{5}}\left(\tilde{x}_{n}=\tilde{a}, \tilde{y}_{n}\right)=\left(\frac{b\left(a-c q^{4}\right)\left(a-d q^{4}\right)}{(a-b)\left(a+b-c q^{3}-d q^{3}\right)}, a\right)
$$

(iii) If $\tilde{x}_{n}=\tilde{b}$ and $(a-b)(a+b-c q-d q) \tilde{y}_{n} \not \equiv-a(b-c)(b-d)$, by an argument similar to that in (i), we have

$$
\widetilde{\Phi_{n}^{3}\left(x_{n}, y_{n}\right)}=\widetilde{\Phi_{n}^{3}}\left(\tilde{x}_{n}=\tilde{b}, \tilde{y}_{n}\right)=\left(\frac{b\left(a-c q^{2}\right)\left(a-d q^{2}\right) \tilde{y}_{n}}{a(b-c)(b-d)+(a-b)(a+b-c q-d q) \tilde{y}_{n}}, a\right) .
$$

(iv) If $\tilde{x}_{n}=\tilde{b}$ and $(a-b)(a+b-c q-d q) \tilde{y}_{n} \equiv-a(b-c)(b-d)$, by an argument similar to that in (ii), we have

$$
\widetilde{\Phi_{n}^{5}\left(x_{n}, y_{n}\right)}=\widetilde{\Phi_{n}^{5}}\left(\tilde{x}_{n}=\tilde{b}, \tilde{y}_{n}\right)=\left(-\frac{a\left(b-c q^{4}\right)\left(b-d q^{4}\right)}{(a-b)\left(a+b-c q^{3}-d q^{3}\right)}, b\right)
$$

(v) If $\tilde{y}_{n}=0$ and $\tilde{x}_{n} \neq 0$,

$$
\Phi_{n}^{3\left(x_{n}, y_{n}\right)}=\widetilde{\Phi_{n}^{3}}\left(\tilde{x}_{n}, \tilde{y}_{n}=0\right)=\left(0, \frac{a b}{\tilde{x}_{n}}\right) .
$$

(vi) If $\tilde{y}_{n}=0$ and $\tilde{x}_{n}=0$,

$$
\widetilde{\Phi_{n}^{4}\left(x_{n}, y_{n}\right)}=\widetilde{\Phi_{n}^{4}}\left(\tilde{x}_{n}=0, \tilde{y}_{n}=0\right)=(0,0) \text {. }
$$

We have now fully investigated the behaviours around singularities and have completed the proof. 


\section{$3.2 q$-discrete Painlevé IV equation}

The $q$-discrete analogue of Painlevé IV equation has the following form

$$
\left(x_{n+1} x_{n}-1\right)\left(x_{n} x_{n-1}-1\right)=\frac{a q^{2 n}\left(x_{n}^{2}+1\right)+b q^{2 n} x_{n}}{c x_{n}+d q^{n}},
$$

where $a, b, c, d$ and $q$ are parameters $[10,11]$. It can be rewritten as follows:

$$
\Phi_{n}:\left\{\begin{array}{l}
x_{n+1}=\frac{\tau^{2}\left(a x_{n}^{2}+b x_{n}+a\right)+\left(x_{n} y_{n}-1\right)\left(x_{n}+\tau\right)}{x_{n}\left(x_{n} y_{n}-1\right)\left(x_{n}+\tau\right)} \\
y_{n+1}=x_{n},
\end{array}\right.
$$

where $\tau=q^{n} \tau_{0}$. Here we took $\tau_{0}=d / c$ and redefined $a, b$ as $a c / d^{2} \rightarrow a$ and $b c / d^{2} \rightarrow b$.

Proposition 3.2. Suppose that $|a|_{p}=|b|_{p}=|q|_{p}=\left|\tau_{0}\right|_{p}=1$, and we also suppose that $a q^{2} \tau_{0} \not \equiv 1$ and $a q^{4} \tau_{0} \not \equiv 1$. Then the mapping (3) has almost good reduction modulo $\mathfrak{p}$ on the domain $\mathcal{D}^{(n)}:=\left\{(x, y) \in \mathbb{Z}_{p}^{2} \mid x \neq 0, x y \neq 1, x \neq-q^{n} \tau_{0}\right\}$.

Proof. In the proof we use the abbreviation as $\tilde{a} \rightarrow a, \tilde{b} \rightarrow b, \tilde{\tau}_{0} \rightarrow \tau_{0}$. By an argument similar to that in Proposition 3.1, we have only to consider the cases at the singular points modulo a prime.

(i) If $\tilde{x}_{n}=0$ and $1+q^{2}\left(-1+a \tau_{0}-b \tau_{0}^{2}+q \tau_{0}^{2}+\tau_{0} y_{n}-a \tau_{0}^{2} y_{n}\right) \not \equiv 0$, the first and second iterations, $\widetilde{\Phi_{n}}\left(0, \tilde{y}_{n}\right)$ and $\widetilde{\Phi_{n}^{2}}\left(0, \tilde{y}_{n}\right)$ are not well-defined. However, at the third iteration we have

$$
\begin{aligned}
\widetilde{\Phi_{n}^{3}\left(x_{n}, y_{n}\right)} & =\widetilde{\Phi_{n}^{3}}\left(\tilde{x}_{n}=0, \tilde{y}_{n}\right) \\
& =\left(\frac{-1-q^{3} \tau_{0}^{2}-b q^{4} \tau_{0}^{2}+a q^{6} \tau_{0}^{3}+q^{2}\left(1+b \tau_{0}^{2}-\tau_{0} \tilde{y}_{n}+a \tau_{0}^{2} \tilde{y}_{n}\right)}{q^{2} \tau_{0}\left\{1+q^{2}\left(-1+a \tau_{0}-b \tau_{0}^{2}+q \tau_{0}^{2}+\tau_{0} \tilde{y}_{n}-a \tau_{0}^{2} \tilde{y}_{n}\right)\right\}},-q^{2} \tau_{0}\right) .
\end{aligned}
$$

(ii) If $\tilde{x}_{n}=0$ and $1+q^{2}\left(-1+a \tau_{0}-b \tau_{0}^{2}+q \tau_{0}^{2}+\tau_{0} y_{n}-a \tau_{0}^{2} y_{n}\right) \equiv 0$, we iterate the map further from (i) until the reduced map is well-defined at $\tilde{y}_{n}=\frac{1+q^{2}\left(-1+a \tau_{0}-b \tau_{0}^{2}+q \tau_{0}^{2}\right)}{q^{2} \tau_{0}\left(a \tau_{0}-1\right)}$. At the fifth iteration,

$$
\left.\Phi_{n}^{5} \widetilde{\left(x_{n}, y_{n}\right.}\right)=\widetilde{\Phi_{n}^{5}}\left(\tilde{x}_{n}=0, \tilde{y}_{n}\right)=\left(\frac{-1+q^{2}+a q^{4} \tau_{0}+q^{7} \tau_{0}^{2}-b q^{8} \tau_{0}^{2}}{q^{4} \tau_{0}\left(-1+a q^{4} \tau_{0}\right)}, 0\right)
$$

Since we assumed that $a q^{4} \tau_{0} \not \equiv 1$, it is well-defined.

(iii) If $\tilde{x}_{n}=-q^{n} \tau_{0}$ and $\tilde{y}_{n} \neq-\tau_{0}^{-1}$,

$$
\begin{aligned}
& \Phi_{n}^{3\left(x_{n}, y_{n}\right)}=\widetilde{\Phi_{n}^{3}}\left(\tilde{x}_{n}=-q^{n} \tau_{0}, \tilde{y}_{n}\right) \\
& \quad=\left(\frac{-1-\tau_{0} \tilde{y}_{n}+\left(q^{3}-b q^{4}\right) \tau_{0}^{2}\left(1+\tau_{0} \tilde{y}_{n}\right)+q^{2}\left\{1+b \tau_{0}^{2}+\tau_{0} \tilde{y}_{n}+a \tau_{0}^{2}\left(-\tau_{0}+\tilde{y}_{n}\right)\right\}}{q^{2} \tau_{0}\left(-1+a q^{2} \tau_{0}\right)\left(1+\tau_{0} \tilde{y}_{n}\right)}, 0\right),
\end{aligned}
$$

where we assumed $a q^{2} \tau_{0} \not \equiv 1$.

(iv) If $\tilde{x}_{n}=-q^{n} \tau_{0}$ and $\tilde{y}_{n}=-\tau_{0}^{-1}$,

$$
\widetilde{\left.\Phi_{n}^{5} \widetilde{\left(x_{n}, y_{n}\right.}\right)}=\widetilde{\Phi_{n}^{5}}\left(\tilde{x}_{n}=-q^{n} \tau_{0}, \tilde{y}_{n}=-\tau_{0}^{-1}\right)=\left(-\frac{1}{a q^{6} \tau_{0}^{2}},-a q^{6} \tau_{0}^{2}\right) .
$$

(v) If $\tilde{x}_{n} \tilde{y}_{n}=1$,

$$
\left.\Phi_{n}^{5} \widetilde{\left(x_{n}, y_{n}\right.}\right)=\widetilde{\Phi_{n}^{5}}\left(\tilde{x}_{n}=\frac{1}{\tilde{y}_{n}}, \tilde{y}_{n}\right)=\left(\frac{1}{a q^{6} \tau_{0}^{3} \tilde{y}_{n}}, a q^{6} \tau_{0}^{3} \tilde{y}_{n}\right) .
$$




\section{Hietarinta-Viallet equation over a finite field}

The Hietarinta-Viallet equation [5] is the following difference equation:

$$
x_{n+1}+x_{n-1}=x_{n}+\frac{a}{x_{n}^{2}},
$$

with $a$ as a parameter. The equation (4) passes the singularity confinement test [3], which is a notable test for integrability of equations, yet is not integrable in the sense that its algebraic entropy [1] is positive and that the orbits display chaotic behaviour [5, 17]. We prove that the AGR is satisfied for this Hietarinta-Viallet equation. We rewrite (4) as the following coupled system:

$$
\Phi_{n}:\left\{\begin{array}{l}
x_{n+1}=x_{n}+\frac{a}{x_{n}^{2}}-y_{n}, \\
y_{n+1}=x_{n} .
\end{array}\right.
$$

Proposition 4.1. Suppose that $|a|_{p}=1$, then the mapping (5) has almost good reduction modulo $\mathfrak{p}$ on the domain $\mathcal{D}:=\left\{(x, y) \in \mathbb{Z}_{p}^{2} \mid x \neq 0\right\}$.

Proof. If $\tilde{x}_{n} \neq 0$ then, the next step is immediately well-defined: $\widetilde{\Phi_{n}\left(x_{n}, y_{n}\right)}=\widetilde{\Phi_{n}}(\tilde{x}, \tilde{y})$.

If $\tilde{x}_{n}=0$, we have to iterate the map four times to obtain

$$
\widetilde{\Phi_{n}^{4}\left(x_{n}, y_{n}\right)}=\widetilde{\Phi_{n}^{4}}\left(\tilde{x}_{n}=0, \tilde{y}_{n}\right)=\left(\tilde{y}_{n}, 0\right) .
$$

Therefore we learn that the AGR works similarly to the singularity confinement test in distinguishing the integrable systems from the non-integrable ones. In fact, the AGR can be seen as an arithmetic analogue of the singularity confinement test.

\section{Concluding remarks}

We studied the integrable discrete equations over a finite field by reducing them from the field of $p$-adic numbers. We considered the "almost good reduction" (AGR), which had been proposed to be closely related to the integrability of discrete dynamical systems over finite fields. We proved that $q$-discrete Painlevé III and IV equations also have AGR, which has been a conjecture in our previous article. We also treated the Hietarinta-Viallet equation, which is non-integrable yet passes singularity confinement test. We proved that it also has AGR. From these observations, we have concluded that AGR is not a complete integrability test, however, we can safely state that the AGR is an arithmetic dynamical analogue of the singularity confinement method. One of the future problems is to modify AGR so that it can identify the systems which are nonintegrable yet pass the singularity confinement test, like the Hietarinta-Viallet equation. Other future problems are listed below: (i) to study the geometric construction of the initial value space by blowups of the projective space of $\mathbb{Q}_{p}$, and its relation to the Sakai's theory [14], (ii) to study the relation of our methods to the algebraic entropy [1] and its arithmetic analogue [4], (iii) to formulate the properties of the reduction modulo prime of the higher dimensional mappings like those in [6], (iv) to extend our methods to lattice equations with soliton solutions, such as the discrete Korteweg-de Vries equation and the discrete nonlinear Schrödinger equation.

\section{Acknowledgements}

The author wish to thank Professors Jun Mada, K.M. Tamizhmani, Tetsuji Tokihiro and Ralph Willox for insightful discussions and comments. He also thanks the detailed suggestions by the referees. This work is supported by Grant-in-Aid for JSPS Fellows (24-1379). 


\section{References}

[1] Bellon M.P., Viallet C.M., Algebraic entropy, Comm. Math. Phys. 204 (1999), 425-437, chao-dyn/9805006.

[2] Białecki M., Doliwa A., The discrete KP and KdV equations over finite fields, Theoret. and Math. Phys. 137 (2003), 1412-1418, nlin.SI/0302064.

[3] Grammaticos B., Ramani A., Papageorgiou V., Do integrable mappings have the Painlevé property?, Phys. Rev. Lett. 67 (1991), 1825-1828.

[4] Halburd R.G., Diophantine integrability, J. Phys. A: Math. Gen. 38 (2005), L263-L269, nlin.SI/0504027.

[5] Hietarinta J., Viallet C., Singularity confinement and chaos in discrete systems, Phys. Rev. Lett. 81 (1998), 325-328, solv-int/9711014.

[6] Kajiwara K., Noumi M., Yamada Y., Discrete dynamical systems with $W\left(A_{m-1}^{(1)} \times A_{n-1}^{(1)}\right)$ symmetry, Lett. Math. Phys. 60 (2002), 211-219, nlin.SI/0106029.

[7] Kanki M., Mada J., Tamizhmani K.M., Tokihiro T., Discrete Painlevé II equation over finite fields, J. Phys. A: Math. Theor. 45 (2012), 342001, 8 pages, arXiv:1206.4456.

[8] Kanki M., Mada J., Tokihiro T., The space of initial conditions and the property of an almost good reduction in discrete Painlevé II equations over finite fields, arXiv:1209.0223.

[9] Quispel G.R.W., Roberts J.A.G., Thompson C.J., Integrable mappings and soliton equations, Phys. Lett. A 126 (1988), 419-421.

[10] Ramani A., Grammaticos B., Discrete Painlevé equations: coalescences, limits and degeneracies, Phys. A 228 (1996), 160-171, solv-int/9510011.

[11] Ramani A., Grammaticos B., Hietarinta J., Discrete versions of the Painlevé equations, Phys. Rev. Lett. 67 (1991), 1829-1832.

[12] Roberts J.A.G., Order and symmetry in birational difference equations and their signatures over finite phase spaces, in Proceedings of the Workshop Future Directions in Difference Equations, Colecc. Congr., Vol. 69, Univ. Vigo, Serv. Publ., Vigo, 2011, 213-221.

[13] Roberts J.A.G., Vivaldi F., Signature of time-reversal symmetry in polynomial automorphisms over finite fields, Nonlinearity 18 (2005), 2171-2192.

[14] Sakai H., Rational surfaces associated with affine root systems and geometry of the Painlevé equations, Comm. Math. Phys. 220 (2001), 165-229.

[15] Silverman J.H., The arithmetic of dynamical systems, Graduate Texts in Mathematics, Vol. 241, Springer, New York, 2007.

[16] Takahashi Y., Irregular solutions of the periodic discrete Toda equation, Master's thesis, The University of Tokyo, 2011 (in Japanese).

[17] Takenawa T., Algebraic entropy and the space of initial values for discrete dynamical systems, J. Phys. A: Math. Gen. 34 (2001), 10533-10545, nlin.SI/0103011.

[18] Wolfram S., Statistical mechanics of cellular automata, Rev. Modern Phys. 55 (1983), 601-644. 